\title{
Recognition Without Identification, Erroneous Familiarity, and Déjà Vu
}

\section{Akira R. O'Connor • Chris J. A. Moulin}

\author{
A. R. O’Connor \\ Department of Psychology, Washington University in St. Louis, One Brookings Drive, St. Louis, \\ MO 63130, USA \\ e-mail: aoconnor@wustl.edu
}

\begin{abstract}
Déjà vu is characterized by the recognition of a situation concurrent with the awareness that this recognition is inappropriate. Although forms of déjà vu resolve in favor of the inappropriate recognition and therefore have behavioral consequences, typical déjà vu experiences resolve in favor of the awareness that the sensation of recognition is inappropriate. The resultant lack of behavioral modification associated with typical déjà vu means that clinicians and experimenters rely heavily on self-report when observing the experience. In this review, we focus on recent déjà vu research. We consider issues facing neuropsychological, neuroscientific, and cognitive experimental frameworks attempting to explore and experimentally generate the experience. In doing this, we suggest the need for more experimentation and a more cautious interpretation of research findings, particularly as many techniques being used to explore déjà vu are in the early stages of development.
\end{abstract}

Keywords Memory, Déjà vécu, Dissociation, Recollection, Confabulation 


\section{Introduction}

The sensation of déjà vu arises as a conjunction of two streams of cognition: the phenomenological experience of recognizing a current situation and the awareness that this feeling of recognition is inappropriate. Most importantly, the overall evaluation of the déjà vueliciting situation sides with the higher-order metacognitive awareness of inappropriate recognition - the outcome is that the experient is able to function normally, does not modify his or her behavior based on the errant sense of recognition, and can be left with a sense of wonderment at this insight into the normally concealed machinations of his or her mnemonic decision-making processes. Déjà vu is therefore a benign experience, not a pathological one, as it does not lead to a behavioral impairment. It is only in related déjà experiences, such as recollective confabulation (see below), that we see behavioral evidence of a metacognitive impairment in the form of altered behavior that accommodates the sensation of errant recognition. In this review, we provide an overview of recent déjà vu research focusing on: 1) déjà experiences in clinical groups and the affordances of neuropsychological and neuroscientific methods with which they are typically investigated and 2) the burgeoning field of déjà vu research on healthy populations.

The review and book by Brown $[1,2]$ shone the spotlight on déjà vu and helped catalyze research output in the field. Seven years on, we aim to provide an overview of what we have learned thus far from neuropsychological, neuroscientific, and psychological research into déjà experiences, and the pitfalls facing empiric research of an inherently subjective phenomenon. These pitfalls are special considerations that ideally should be made when dealing with déjà vu research in clinical and nonclinical populations. These special considerations fall into two broad 
domains: the first domain recognizes the way in which our conceptualization of functional neuroanatomy has changed with recent advances in neuroimaging; the second outlines social psychological principles that must be considered when observing self-reported phenomena. Both are important in untangling the sometimes tenuous relationship between intervention and observation with regard to déjà vu and related memory phenomena.

\section{Neuropsychology and Neuroscience}

Déjà vu research has its origins in neuropsychology [3]. Traditional case studies and then brain stimulation studies paved the way for the modern neuroscientific conceptualization of déjà vu as being associated (if not causally intertwined) with seizures and epilepsy [4, 5]. Although the clinically oriented case study has even recently made up the bulk of the déjà vu literature output [6-9], there is a growing trend toward the use of modern neuroscience techniques to make inferences about brain activation that is associated with the occurrence of déjà vu experiences. Herein we describe some of the more recent clinically oriented neuropsychology and neuroscience articles concerning déjà vu, with particular emphasis on 1) the discussion of brainbased inference and 2) a new model that proposes a neurological mechanism for the condition of déjà vécu.

Brain-based Inference and Déjà Vu

Typical case studies report a neuropathology (often epilepsy or dementia, although it can also be drug use, migraine, etc.) that greatly elevates the occurrence of déjà vu in the affected individual. 
Lee et al. [10] similarly reported the case of a teenager who presented with seizures and déjà vu and olfactory auras that resolved following amygdalectomy. They reported that this is a demonstration that amygdala pathology alone (as opposed to in concert with other mesial temporal lobe structures) is capable of generating auras of this nature.

Kovacs et al. [11] provided a comprehensive account of the treatment of hemidystonia with deep brain stimulation (DBS) to the globus pallidus. What differentiates this account from typical stimulation studies is that on noticing DBS-elicited déjà vu, the authors followed up with single photon emission CT imaging comparing DBS leading to déjà vu with DBS not leading to déjà vu. They found DBS-evoked déjà vu to be associated with hyperperfusion in the right hemisphere mesial temporal structures.

Both reports selectively implicate the mesial temporal regions in the generation of déjà vu. This is in keeping with the previous literature, including reports that mesial activation spreading to the neocortex can even inhibit the generation of déjà vu [12•]. Despite these findings, and aside from straightforward problems of brain-behavior inference [13], it would be unwise to consider that these mesial temporal structures in isolation can be responsible for the sensation of déjà vu. There is a growing interest in functional connectivity—slow-wave covariation of activation within discrete networks that is also independent of activation in other networks - that suggests the traditional, neuropsychology-driven, modular view of functional architecture (eg, hippocampus $=$ recollection center, prefrontal cortex $=$ cognitive control center $)$ is incomplete $[14,15]$. Even modular units such as the hippocampus, whose lesion results in a very clear deficit, are now being reconceptualized as lying within far broader networks [16], whereas regions whose involvement in higher-order cognition was not previously considered, such as the cerebellum, are being implicated in networks associated with functions such as 
cognitive control [17]. Thus, aberrant localized activation resulting in a particular experience must be considered in relation to a number of factors additional to the intuitive argument that because activation in region $\mathrm{X}$ has been elevated compared with baseline, region $\mathrm{X}$ alone must be responsible for the activation-induced experience A.

Four additional factors that should be considered when suggesting a causal relationship between aberrant localized activation and déjà vu are as follows: 1) spreading localized activation, 2) spreading functional network activation, 3) the functional network region in which activation is localized, and 4) the congruence of the localized activation with the activation in the rest of the functional network. Spreading localized activation is most frequently considered by those reporting stimulation studies. Discharge to regions $\mathrm{Y}$ and $\mathrm{Z}$, which neighbor region $\mathrm{X}$, is considered as a potential cause of experience $\mathrm{A}$ in much the same way as region $\mathrm{X}$ alone, although this factor once again fails to consider the overarching role of the functional connectivity network. Spreading functional network activation provides the opportunity for a more distributed set of nodes (eg, regions Q, L, and F), which may be located in areas distant from region X, to be responsible for experience A (as globus pallidus stimulation caused contralateral mesial temporal activation in the discussed Kovacs et al. [11] article), with further consideration of the temporal dynamics of this spreading activation introducing another point at which activation may deviate from nonaberrant activation [18]. The functional network region in which activation is localized is important to consider in light of findings that the displacement of functional connectivity "seed" regions by millimeters, even within the same brain structure, can lead to the identification of vastly different networks or the identification of differing regions on the borders of the same functional network $[19,20]$. This is perhaps most salient when comparing activation and experiences resulting from artificial electrical stimulation in separate 
but closely spaced regions and should be considered together with the previous two factors. Finally, the congruence of the localized activation with the activation in the rest of the functional network is potentially the most nuanced consideration. Recent studies have found that different patterns of intra-network correlations are associated with different behavioral response states [20, 21]. Aberrant activation of region $X$ may therefore differ not only when compared with baseline activation but also when compared with the pattern of activation within other regions of the functional network. Within such a framework, the duality of the déjà vu experience is intuitively plausible; mesial temporal structures may aberrantly indicate a sensation of familiarity despite the rest of the hippocampo-cortical network indicating the overarching nonrecognition state that ultimately presides.

These additional considerations complicate an otherwise-straightforward story of activation-based causality. However, we believe they reflect a more realistic interpretation of the multitude of interconnections that characterize our on- and off-task cognitions. Therefore, we suggest that future neuroscientific investigation of déjà vu be mindful of current understandings of functional architecture, which may also require further investigation within the clinical samples in question.

\section{Recollective Confabulation: Déjà Vécu}

Attempts have been made to subcategorize the déjà vu experience, the outcomes of which are various French past participles, such as déjà entendu or déjà visité, which refer to the event or modality triggering the experience. However, little or no consensus exists for these different terms, and there is scant empiric support. The only discrimination of possible clinical relevance 
is of déjà vu versus déjà vécu, being that it is a theoretically plausible distinction based on a case series of patients with neurological damage $[9,22]$.

Contemporary memory theory posits that two separable processes are associated with separate neural structures $[23,24]$. In short, these two processes map to two different phenomenological states: recollection and familiarity. For déjà vu, an inappropriate feeling of familiarity is experienced (alongside the awareness that it is inappropriate), whereas for déjà vécu, the sensation derives from false feelings of recollection (as outlined subsequently, not necessarily alongside the awareness that it is inappropriate). In this experience, instead of a vague feeling of familiarity for some materials, the experient senses a more complete sensation of retrieval of the information, including contextual details, a sense of mental time travel, and the sensation that he or she knows what will happen next.

The evidence from déjà vécu comes from a number of cases, typically older adults with dementia [9]. Caution is required in the classification of déjà vécu in all groups because the primary evidence for this state comes from patients who are largely anosognosic. These patients tend to act on their sensation of false recollection; thus, the clash at the core of the experience is not present. Déjà vécu episodes are often associated with novel events and experiences and are typically justified by the experient in a confabulatory manner (termed recollective confabulation) [25•]. The recollective nature of the déjà vécu experience is incongruous with the benign (as far as memory decision making is concerned) nature of the typical déjà vu experience, which has been investigated in the context of familiarity. However, this recollective experience dissociation may actually help us better understand the causal mechanisms underpinning déjà vécu.

A recent article in Cognitive Neuropsychiatry proposed a mechanism for déjà vécu by which an erroneous sense of recollection could be experienced for experiences and situations that 
should be interpreted as novel [26••]. This mechanism hinges on two sets of findings: first that the hippocampus is involved in signaling both recollection [27] and novelty [28], and second that depending on whether these cells are primarily encoding (during novelty) or retrieving information (during recollection), they fire at different mean phases of the hippocampal theta oscillation [29]. According to this mechanism, in déjà vécu, regions downstream of the hippocampus are disrupted such that retrieval-responsive regions become responsive to signals with a mean theta phase similar to that associated with the hippocampal-encoding signal. Thus, in déjà vécu (and aside from any concurrent impairments), novel stimuli that elicit false recollection should at some point in the functional network become associated with a thetadephased signal that has a similar mean theta phase to that which signals recollection.

A precise mechanism for theta dephasing is not proposed, but it is conceivable that in clinical pathologies associated with déjà vécu, ample opportunity exists for this dephasing to occur, thereby generating the erroneous recollection instead of novelty. Most importantly, this mechanism provides testable hypotheses that should be observable using electrophysiologic recording (though not functional MRI): 1) novel stimuli that generate déjà vécu should elicit neocortical firing within the hippocampo-cortical network with a similar phase to the firing elicited by true recollection and 2) novel stimuli that do not generate déjà vécu should elicit neocortical firing within the same network with a different phase to the firing elicited by true recollection (and also to déjà vécu). These hypotheses remain to be tested in clinical populations, and many difficulties may be encountered in doing this given the invasive nature of electrophysiologic recording. Even if the model is supported by evidence from those with déjà vécu, the validity of its extension to déjà vu, given the previously outlined differences, is questionable. Nevertheless, this model provides a theoretically driven framework that lends itself 
to empiric validation. This is something that is lacking in the neuroscientific research of clinically mediated déjà experiences as a whole, and presents the field with exciting possibilities for the understanding—as opposed to just the observation—of the déjà experience.

Psychopathology and Déjà Vu

The cognitive neuropsychiatry approach outlined previously advocates understanding psychiatric conditions as well as neurological conditions from an information processing framework and explores nebulous sensations such as delusion and false memory in psychiatric conditions such as schizophrenia. It is possible that the understanding of déjà vu could be improved by the study of such conditions. Interestingly, a large survey of schizophrenia patients showed that they actually experienced déjà vu less frequently than control participants, although the schizophrenia patients reported being more distressed by the experience [30•].

Until recently, clinical occurrences of déjà vu were often described interchangeably with depersonalization phenomena [31]. However, the core cognitive neuropsychiatric concept heredissociation-and déjà vu experience do not seem to be related in healthy populations [32], and anxiety disorder patients with or without depersonalization and derealization symptoms experienced déjà vu and déjà vécu to equal degrees: between one third and one half of all patients studied [33]. In sum, déjà vu may not be seen as a meaningful symptom in psychiatry, although the response to it by schizophrenia patients may be more pronounced, and certain groups tend to experience it more often than healthy groups (anxiety disorders, people with derealization/depersonalization). Because a neurobiological model of depersonalization exists [34], invoking the same limbic-temporal networks reviewed in the epilepsy section above, it 
seems that exploration of the déjà state in depersonalization could be a worthwhile avenue for future research.

Summary: Neuropsychology and Neuroscience

Déjà vu and déjà vécu research in clinical populations continues to provide insight into the possible neurological antecedents of déjà experiences. Research on clinical groups typically affords the clinician access to neuroscientific methods that would be nonviable in nonclinical populations who experience déjà vu far less frequently or have no reason to subject themselves to invasive neurophysiologic procedures. Although invasive neuroscientific methods within clinical groups have inferential power beyond standard functional neuroimaging methods, it is worth considering that our understanding of brain systems has progressed to conceptualize functional networks whose intra- and internetwork relationships may be important in shaping the phenomenological experience above and beyond the traditional modular conceptualization of brain activation. With this conceptualization in mind, a novel model of déjà vécu, informed by behavioral neuroscience, may yield support from clinical groups with déjà vécu. However, comparisons between clinical and nonclinical varieties of déjà vu are potentially troublesome given the phenomenological and behavioral differences associated with the comparison of the two experiences. Therefore, it is fitting to review the psychological literature on déjà vu in the nonclinical population, particularly as there has been tremendous growth in this form of research in recent years.

\section{Experimental Cognitive Psychology}


Studying clinical groups can maximize the likelihood that the researcher will encounter people who are very aware of the intricacies of their déjà experiences, but these experiences may be somewhat different from those not associated with an underlying neurological condition. Therefore, it is necessary to complement the study of déjà vu and déjà vécu in clinical groups with the study of the nonclinical population. Before we review this literature, it is necessary to resolve a few conceptual and definitional issues at the heart of the subjectivity of the nonclinical déjà vu experience.

The subjective nature of the typical déjà vu experience lies in its one-sided resolution toward unfamiliarity; an individual feels that he or she has visited a place before but knows that he or she has not and acts accordingly. In a laboratory setting on a standard memory task, its occurrence cannot be inferred without asking the experient whether during the previous moments when he or she produced a series of perfectly acceptable answers to a number of "old/new" recognition questions, the experient also happened to experience déjà vu. That is, there is no behavioral index of déjà vu. This is markedly different from other memory phenomena, such as false recognition resulting from the Deese-Roediger-McDermott procedure [35], which is evidenced by remarkable patterns of responding, but about whose occurrence experients are none the wiser. As a consequence, we cannot point to unusual patterns of response in memory tests to validate the occurrence of the sensation but must instead ensure that the way in which we have asked potential experients to examine their cognitive processes is precise enough to avoid false alarms. This emphasis on the avoidance of type I error is particularly important, as prior to the publication of the seminal review and book by Brown [1,2], only one series of studies had reported a noninvasive procedure capable of generating a sensation akin to déjà vu (and even 
then, the authors were extremely careful in their wording with regard to déjà vu, preferring the term restricted paramnesia) [36, 37]. With the experimental interrogation of déjà vu in its infancy, those who wish to generate and measure the sensation must be prudent in their assertions of its generation and causes, and even more careful in any inferences derived from these assertions. In short, déjà vu is not the same as a false memory experience. Many paradigms and memory illusions in the laboratory give rise to false memory, strange feelings of familiarity, and dissociations between conscious and nonconscious behaviors, and if (as is usually the case) the phenomenological experience of recognition is taken as indicative of a bona fide memory and does not lead to the clash of evaluations at the heart of déjà vu, then this experience is a false memory and not a déjà vu experience. As a case in point, if one goes to a novel place but finds it familiar without contesting this internal assessment, he or she will believe that he or she has actually been there before and will not be experiencing déjà vu. We return to this issue in the paradigms described below.

Invasive methods of déjà vu generation abound. These include reliable methods such as electrical brain stimulation $[4,5,38]$ and caloric stimulation (the flushing of ear canals with water) [39] and less reliable but nonetheless compelling methods such as the ingestion of certain drugs $[40,41]$. In contrast, comparatively little success has been achieved in generating déjà vu by noninvasive, experimental methods. However, experiments that can be categorized as falling into one of two noninvasive experimental methods-familiarity through prior exposure and hypnotic suggestion—-have been reported in recent years.

Both familiarity-based and hypnotic methods are intended to provide the conditions necessary for the experience of déjà vu. However, these methods can make markedly different assumptions of the presupposed antecedents of the experience. This has been discussed as the 
difference between data-driven and higher-order conceptualizations [7] and can be distilled down to whether déjà vu is generated as a result of an appropriate sensation of familiarity that feels inappropriate due to a lack of other contextualizing recognition (data driven), or whether it results from an overarching sense of inappropriate familiarity that is applied to everything in the experient's perceptual stream (higher order). This debate remains unresolved, although it is interesting to note that cognitive experimental theorists tend to presuppose a data-driven conceptualization, whereas the invasive methods outlined previously lend themselves to explanation by a higher-order conceptualization.

Familiarity-based Methods

Three recent articles drawing on traditional cognitive psychological methods for the measurement of recognition have reported the generation of déjà vu. These accounts attribute participant-reported déjà vu to restricted activation of memory traces resulting from prior exposure, seating themselves firmly within the data-driven conceptualization of the phenomenon. In the first article reviewed here, Cleary and Reyes [42] explored earlier reports of déjà vu during a recognition without identification (RWI) paradigm. Participants were presented with the names of famous places at study and identified scenes, some of which corresponded to the previously studied place names, at test. Cleary and Reyes [42] focused primarily on their RWI effects, although what seems remarkable about the procedure is that it led to $87 \%$ of participants (33 of 38) reporting at least one incidence of resultant déjà vu. This finding is extraordinary for several reasons. First, this experimental generation of déjà vu seems almost as reliable as the generation of tip-of-the-tongue state (the subjective experience of almost being able to retrieve a particular 
memory trace but being unable to do so for the duration of the experience, reported by $97 \%$ of participants in the same experiment), which is a near-universal experience, occurs in a nonexperimental setting about once per week, and is known to be reliably generated by certain experimental procedures [43]. Second, our unpublished observations indicate that $84 \%$ of a sample of students at Leeds University (190 of 206) reported having had at least one déjà vu experience in the previous 6 months; this procedure appears on par with 6 months of nonexperimental experience (or to far exceed it if the number of individual déjà vu experiences reported are taken into account) in generating déjà vu. Finally, despite its high occurrence in the procedure by Cleary and Reyes [42], the déjà vu generation is not reported in the multitude of studies eliciting familiarity without recollection published each year. These inconsistencies are puzzling.

Although it is possible that RWI and similar procedures do reliably generate déjà vu, we suspect that reports of déjà vu in $87 \%$ of participants may be an artifact of the way in which déjà vu occurrence was assessed.

An unfortunate problem with the assessment of déjà vu is that the term has become so diluted as to indicate the occurrence of the same (or similar) situation more than once, even within the scientific literature [25•]. As a consequence, researchers must provide an operational definition of the term for participants prior to its assessment, something that Cleary and Reyes [42] did with the following text:

"A déjà vu state means that you are experiencing a vivid feeling that you have experienced something before, even when you know you haven't. For example, you may enter a room and experience déjà vu, such that you feel like you have entered or seen that particular room sometime in the past, even when you know you have never been there before." 
The strongest criticism of this definition is that it is ambiguous as to whether déjà vu would normally be experienced under the circumstances of RWI. The experience of being unable to name a picture even though you know you have studied the picture in a different modality (scene vs word) satisfies this definition by virtue of the modality shift. Equally, a participant who is unsure of exactly what sort of memory phenomenon he or she is experiencing (which is especially important, as déjà vu and tip of the tongue were assessed between participants) may respond positively when questioned about déjà vu because the sensation is somewhat akin to the feeling that person is having. The criticism that a particular line of questioning may lend itself to demand characteristics is not particularly elegant, but under the circumstances of déjà vu being a subjective phenomenon that has previously proven very difficult to generate in the laboratory, we believe it is critical [44].

The second article from the same laboratory used a recognition without cued recall (RWCR) paradigm to test whether configural similarity in visually presented pictures could generate déjà vu [45•]. The experimenters presented scenes at test that were configurally similar to those presented at study. On an item-by-item basis, participants provided responses to items assessing recall, familiarity, and déjà vu occurrence. In certain subsets across three experiments, participants reported déjà vu for up to $68 \%$ of items. In the conditions of interest in separate experiments, during the presence of RWCR for configurally similar test scenes, participants reported déjà vu for $17 \%$ and 26\% of items (experiments 1 and 2A, respectively). Crucially, however, they also reported déjà vu for $13 \%$ and $23 \%$ of items in the control conditions (experiments 1 and 2A respectively) — test scenes that were dissimilar to the studied scenes and should not have generated déjà vu according to the experimental rationale. These differences of $4 \%$ and $3 \%$ were statistically significant, but the number of déjà vu reports in the control 
conditions suggests that what participants reported as déjà vu may have differed considerably from the construct the experimenter intended to assess. Indeed, in the same article, Cleary and colleagues [45 ${ }^{\circ}$ reported that in an independent sample of 92 participants, only $7 \%$ provided a definition capturing the dissociation between familiarity and awareness that we believe to be crucial to the experience. Moreover, they also found that across their series of experiments, when an operational definition of déjà vu was not provided, participants were more likely to report its occurrence, suggesting that efforts to constrain false-positive reports, when used, can be successful. Cleary and colleagues [45•] summarized that their RWCR procedure elevated the incidence of déjà vu, but that more time must be spent untangling déjà vu from other constructs and confounds. We would go one step further and suggest that procedures such as RWCR and RWI can elevate the incidence of reported déjà vu, but given the widespread misunderstanding of the term déjà $v u$ and its infrequency of spontaneous report in both similar cognitive psychology experiments and the natural setting as a whole, it is the responsibility of the experimenter to establish that these reports correspond to the actual experience of déjà vu.

The last recent article reviewed here, by Brown and Marsh [46•], reported that subliminal processing of unfamiliar symbols (exposure of $35 \mathrm{~ms}$ ) increases the likelihood that following subsequent supraliminal presentation of the same symbols, they are rated as having been seen prior to the experiment. Postexperimental questionnaire reports indicated that $50 \%$ of participants (12 of 24) experienced déjà vu, supporting the conceptualization of déjà vu by Brown and Marsh [46•] as a data-driven experience [47], although this time attributed to double or split perception $[6,48]$. This report provides another method for the generation of déjà vu to explore. It would be particularly interesting to establish whether participants would report the 
normally noteworthy déjà vu experience spontaneously if they were given the opportunity to do so.

With these considerations in mind, in Table 1, we suggest ways in which studies may attempt to protect themselves from the criticism of artifact. The preferred methods suggested are potentially unwieldy (eg, qualitative responses, a multi-item postexperimental questionnaire), but we believe that it is currently important to establish the degree to which experimentally generated experiences of déjà vu resemble the elusive (and rather infrequent) naturally occurring experience. These safeguards will help establish procedures as effective or not and will thereafter become less necessary. We view them as short-term hurdles that will help to validate potentially groundbreaking experimental procedures, ensuring that further long-term investigation does not result in a great deal of investment (of both time and money [eg, with neuroimaging methods, clinic hours ]) in phenomena in which the experimenters have little interest.

Hypnotic Methods

To the authors' knowledge, the only noninvasive procedure to generate déjà vu-like experiences in the 20th century was carried out by Banister and Zangwill [36]. They used hypnotic presentation of visual stimuli followed by suggestions of posthypnotic amnesia. The subsequent representation of the visual stimuli elicited a reaction from two of five participants that Banister and Zangwill [36] described as "spontaneous reference to descriptions of déjà vu." This procedure was recently revived and updated to reflect current methods in hypnosis by O'Connor et al. [49•]. In addition to suggesting posthypnotic amnesia for a previously encountered puzzle game, O'Connor et al. [49•] suggested posthypnotic familiarity for the game to a separate group 
of participants who had not previously encountered it. Using a series of postexperimental validation checks (adhering to the guidelines outlined in Table 1), they were then able to compare the respective efficacies of the amnesia and familiarity suggestions in generating reports of déjà vu when participants were later presented with the task.

O’Connor et al. [49•] found that both posthypnotic amnesia and familiarity suggestions were successful in generating déjà vu in participants, although they led to qualitatively different experiences. Amnesia suggestions led to reports of déjà vu in three of six participants, although these experiences were characterized by participant reports of source amnesia and confusion. Familiarity suggestions, on the other hand, led to five of six participants experiencing déjà vu, with subjective reports likening these to naturally occurring experiences. These findings support the previously discussed articles in suggesting that experimental methods based on the bottom-up conceptualization of déjà vu are capable of generating self-reported déjà vu experiences. However, they favor the efficacy of methods based on higher-order conceptualizations (in which the eliciting stimulus has not been encountered previously) due to the spontaneous nature of the way these experiences are likened to and then favorably compared with naturally occurring experiences. Although O'Connor et al. [49•] satisfied the criteria outlined in Table 1 for the minimization of artifact, other criticisms make the study's findings far from conclusive. The most obvious critiques are that hypnosis is capable of generating far more powerful demand characteristics than traditional cognitive psychological methods (although "déjà vu" was never mentioned during the hypnosis procedure), and that the method outlined does not translate well as an ecologically valid explanation for naturally occurring déjà vu experiences [50].

Summary: Experimental Cognitive Psychology 
The number of recent studies assessing the experimental generation of déjà vu in the nonclinical population is encouraging. With appropriate systems in place for the minimization of false alarms, it should be possible to identify and refine procedures capable of providing real insight into this metacognitive phenomenon. These eventual procedures that provide ecologically valid mechanisms by which déjà vu could be generated may be capable of contributing to the understanding of metacognition and memory decision making in the same way that the exploration of tip-of-the-tongue research contributed to the understanding of lexical retrieval processes [43].

\section{Conclusions}

We have reviewed two broad categories of recent déjà vu research: neuropsychological and neuroscientific research on clinical individuals and groups, and experimental cognitive psychological research on the nonclinical population. In both domains, we have urged caution against making hasty inferences that could harm clinical, research, and lay understandings of the déjà vu experience. Whether in the neuroscientific or the experimental domain, we advise that the generation of déjà vu should not be attributed to a cause without carefully examining whether other potential causes and confounds could be contributing to or undermining the reported generation of the experience.

The increasing availability of neuroscientific procedures to clinicians and researchers, as well as the growing understanding of déjà vu that has accompanied the recent upsurge in research points to further improvement in our understanding of the déjà vu experience during the 
coming years. Nevertheless, we believe that the clinical and nonclinical domains could accelerate this improvement by better informing and being informed by each other. For example, we hope that future experimental procedures found to be successful at generating déjà vu within the nonclinical population will go some way toward unifying the experimental literature with the clinical literature. Ultimately, it will be possible to experimentally generate déjà vu in the clinical groups that have thus far contributed to our understanding of déjà experiences such as déjà vécu and thereby gain a further measure of the degree to which the results of experimental procedures overlap with clinical manifestations of déjà vu. Of utmost importance throughout the use of these methods are the personal, phenomenological experiences of the individual. The déjà vu experience lends itself to delicate expression, and it is with acknowledgment of this expression that we will further understand the means by which déjà vu occurs.

Disclosure No potential conflicts of interest relevant to this article were reported.

\section{References}

1. Brown AS: A review of the déjà vu experience. Psychol Bull 2003, 129:394-413.

2. Brown AS: The Déjà Vu Experience. Hove, NY: Psychology Press; 2004.

3. Arnaud FL: Un cas d'illusion du 'déjà vu' ou de 'fausse mémoire' [in French]. Annales Médico-Psychologiques 1896, 3:455-471.

4. Penfield W: The twenty-ninth Maudsley lecture: the role of the temporal cortex in certain psychical phenomena. J Ment Sci 1955, 101:451-465. 
5. Bancaud J, Brunet-Bourgin F, Chauvel P, Halgren E: Anatomical origin of déjà vu and vivid "memories" in human temporal lobe epilepsy. Brain 1994, 117:71-90.

6. O'Connor AR, Moulin CJ: Normal patterns of déjà experience in a healthy, blind male: challenging optical pathway delay theory. Brain Cogn 2006, 62:246-249.

7. O'Connor AR, Moulin CJ: The persistence of erroneous familiarity in an epileptic male: challenging perceptual theories of déjà vu activation. Brain Cogn 2008, 68:144-147.

8. Kalra S, Chancellor A, Zeman A: Recurring déjà vu associated with 5hydroxytryptophan. Acta Neuropsychiatrica 2007, 19:311-313.

9. Moulin CJ, Conway MA, Thompson RG, et al.: Disordered memory awareness: recollective confabulation in two cases of persistent déjà vecu. Neuropsychologia 2005, 43:1362-1378

10. Lee DJ, Owen CM, Khanifar E, et al.: Isolated amygdala neurocysticercosis in a patient presenting with déjà vu and olfactory auras. J Neurosurg Pediatr 2009, 3:538-541.

11. Kovacs N, Auer T, Balas I, et al.: Neuroimaging and cognitive changes during déjà vu. Epilepsy Behav 2009, 14:190-196.

12.• Vignal JP, Maillard L, McGonigal A, Chauvel P: The dreamy state: hallucinations of autobiographic memory evoked by temporal lobe stimulations and seizures. Brain 2007, 130:8899.

In the mold of John Hughlings Jackson's formative work in the field, Vignal et al. examine memory hallucinations associated with cortical stimulation and epileptic seizure. They report that the resulting "dreamy states" associated with activation in mesial temporal structure often include déjà vécu but are less likely to occur if there is an early spread of electrical discharge to the temporal neocortex. 
13. Poldrack RA: Can cognitive processes be inferred from neuroimaging data? Trends Cogn Sci 2006, 10:59-63.

14. Raichle ME: Neuroscience: the brain's dark energy. Science 2006, 314:1249-1250.

(Published erratum appears in Science 2007, 315:187.)

15. Fox MD, Snyder AZ, Vincent JL, et al.: The human brain is intrinsically organized into dynamic, anticorrelated functional networks. Proc Natl Acad Sci U S A 2005, 102:9673-9678. 16. Vincent JL, Snyder AZ, Fox MD, et al.: Coherent spontaneous activity identifies a hippocampal-parietal memory network. J Neurophysiol 2006, 96:3517-3531.

17. Krienen FM, Buckner RL: Segregated fronto-cerebellar circuits revealed by intrinsic functional connectivity. Cereb Cortex 2009, 19:2485-2497.

18. Barbeau E, Wendling F, Régis J, et al.: Recollection of vivid memories after perirhinal region stimulations: synchronization in the theta range of spatially distributed brain areas. Neuropsychologia 2005, 43:1329-1337.

19. Vincent JL, Kahn I, Snyder AZ, et al.: Evidence for a frontoparietal control system revealed by intrinsic functional connectivity. J Neurophysiol 2008, 100:3328-3342.

20. O'Connor AR, Han S, Dobbins IG: The inferior parietal lobule and recognition memory: Expectancy violation or successful retrieval? J Neurosci 2010, 30:2924-2934.

21. Cohen MR, Maunsell JH: Attention improves performance primarily by reducing interneuronal correlations. Nat Neurosci 2009, 12:1594-1600.

22. O'Connor AR, Moulin CJ, Cohen G: Memory and consciousness. In Memory in the Real World, edn 3. Edited by Cohen G, Conway MA. Hove, NY: Psychology Press; 2008:327-356. 23. Aggleton JP, Brown MW: Episodic memory, amnesia and the hippocampal-anterior thalamic axis. Behav Brain Sci 1999, 22:425-444; discussion 444-489. 
24. Yonelinas AP, Otten LJ, Shaw KN, Rugg MD: Separating the brain regions involved in recollection and familiarity in recognition memory. J Neurosci 2005, 25:3002-3008.

25. Moulin CJ, Chauvel P: Déjà vu: insights from the dreamy state and the neuropsychology of memory. In New Frontiers in Applied Memory Research. Hove: Psychology Press; 2010:206236.

In this recent review, the authors explore both clinical and experimental déjà vu research. They emphasize the importance of communication between the two domains, particularly the role that cognitive experimental psychology has in informing the understanding and care of people with pathological manifestations of déjà vu.

26.• O'Connor AR, Lever C, Moulin CJA: Novel insights into false recollection: a model of déjà vécu. Cogn Neuropsychiatry 2010 (in press).

We review cases of déjà vécu and propose a novel, testable theory of déjà vécu formation that is consistent with the behavior exhibited by these patients. We posit that déjà vécu results from a dephasing of novelty signaling such that in regions downstream of this dephasing, novelty is misinterpreted as recollection.

27. Düzel E, Vargha-Khadem F, Heinze HJ, Mishkin M: Brain activity evidence for recognition without recollection after early hippocampal damage. Proc Natl Acad Sci U S A 2001, 98:8101-8106.

28. Nyberg L: Any novelty in hippocampal formation and memory? Curr Opin Neurol 2005, $18: 424-428$.

29. Hasselmo ME, Bodelon C, Wyble BP: A proposed function for hippocampal theta rhythm: separate phases of encoding and retrieval enhance reversal of prior learning. Neural Comput 2002, 14:793-817. 
30. Adachi N, Adachi T, Akanuma N, et al.: Déjà vu experiences in schizophrenia: relations with psychopathology and antipsychotic medication. Compr Psychiatry 2007, 48:592-596. Adachi et al. found that déjà vu is less associated with schizophrenia than traditional conceptualizations of depersonalization would suggest.

31. Brauer R, Harrow M, Tucker GJ: Depersonalization phenomena in psychiatric patients. Br J Psychiatry 1970, 117:509-515.

32. Adachi N, Akanu N, Adachi T, et al.: Déjà vu experiences are rarely associated with pathological dissociation. J Nerv Ment Dis 2008, 196:417-419.

33. Toni C, Cassano GB, Perugi G, et al.: Psychosensorial and related phenomena in panic disorder and in temporal lobe epilepsy. Compr Psychiatry 1996, 37:125-133.

34. Sierra M, Berrios GE: Depersonalization: neurobiological perspectives. Biol Psychiatry 1998, 44:898-908.

35. Roediger HL, McDermott KB: Creating false memories: remembering words not presented in lists. J Exp Psychol Learn Mem Cogn 1995, 21:803-814.

36. Banister H, Zangwill O: Experimentally induced visual paramnesias. Br J Psychol 1941, $32: 30-51$.

37. Banister H, Zangwill O: Experimentally induced olfactory paramnesias. Br J Psychol 1941, 32:155-175.

38. Bartolomei F, Barbeau E, Gavaret M, et al.: Cortical stimulation study of the role of rhinal cortex in déjà vu and reminiscence of memories. Neurology 2004, 63:858-864.

39. Adair JC, Na DL, Schwartz RL, Heilman KM: Caloric stimulation in neglect: evaluation of response as a function of neglect type. J Int Neuropsychol Soc 2003, 9:983-988. 
40. Taiminen T, Jääskeläinen SK: Intense and recurrent déjà vu experiences related to amantadine and phenylpropanolamine in a healthy male. J Clin Neurosci 2001, 8:460-462.

41. Singh S: Adolescent salvia substance abuse. Addiction 2007, 102:823-824.

42. Cleary AM, Reyes NL: Scene recognition without identification. Acta Psychol (Amst) 2009, 131:53-62.

43. Brown AS: A review of the tip-of-the-tongue experience. Psychol Bull 1991, 109:204223.

44. Orne MT: On the social psychology of the psychological experiment: with particular reference to demand characteristics and their implications. Am Psychol 1962, 17:776-783. 45. Cleary AM, Ryals AJ, Nomi JS: Can déjà vu result from similarity to a prior experience? Support for the similarity hypothesis of déjà vu. Psychon Bull Rev 2009, 16:1082-1088. Cleary et al. increased reports of experimentally generated déjà vu using an RWCR procedure and emphasized the role of familiarity in the formation of the experience. They proposed future work to clarify the relationship between déjà vu and familiarity.

46. $\quad$ Brown AS, Marsh EJ: Creating illusions of past encounter through brief exposure. Psychol Sci 2009, 20:534-538.

Brown and Marsh used a subliminal presentation procedure to increase misattributions of previous stimuli exposure to pre-experimental experience. Participants reported confusion and déjà vu when making judgments at test.

47. Brown AS, Marsh EJ: Evoking false beliefs about autobiographical experience. Psychon Bull Rev 2008, 15:186-190.

48. Osborn HF: Illusions of memory. North Am Rev 1884, 138:476-486. 
49.• O'Connor AR, Barnier AJ, Cox RE: Déjà vu in the laboratory: a behavioral and experiential comparison of posthypnotic amnesia and posthypnotic familiarity. Int J Clin Exp Hypn 2008, 56:425-450.

The authors compared data-driven and higher-order conceptualizations of déjà vu using a hypnotic analogue in highly hypnotizable participants. Participants spontaneously reported more compelling déjà vu experiences following posthypnotic familiarity suggestions compared with posthypnotic amnesia suggestions.

50. Orne MT: The nature of hypnosis: artifact and essence. J Abnorm Psychol 1959, 58:277299. 
Table 1 Methods for the assessment of déjà vu

\begin{tabular}{lll}
\hline Preferred method & Nonpreferred method & Justification \\
\hline $\begin{array}{l}\text { Operational definition } \\
\text { unambiguously } \\
\text { differentiates déjà vu from } \\
\text { other likely but non-déjà } \\
\text { vu experiences }\end{array}$ & $\begin{array}{l}\text { No provision of an } \\
\text { operational definition }\end{array}$ & $\begin{array}{l}\text { The lay meaning of the term may differ } \\
\text { from the intended meaning }\end{array}$ \\
\hline $\begin{array}{l}\text { Postexperimental } \\
\text { questionnaire }\end{array}$ & $\begin{array}{l}\text { Item-by-item } \\
\text { questioning }\end{array}$ & $\begin{array}{l}\text { Constant reinforcement that déjà vu may } \\
\text { occur on an item-by-item basis may } \\
\text { suggest to participants that it is an } \\
\text { expected aspect of experimental } \\
\text { experience. However, depending on the } \\
\text { type of analysis used, item-by-item } \\
\text { questioning may be unavoidable }\end{array}$ \\
\hline
\end{tabular}

General (eg, confusion, 1 item assessing déjà vu boredom) to specific (eg, déjà vu, tip of the tongue, jamais $v u$ ) progression in multi-item, postexperimental experience questionnaire

\section{occurrence only}

Provides the opportunity for participants to be discerning in their labeling of experiences (ie, not responding "yes" to déjà vu because it seems similar to the actual experimentally generated experience of familiarity without recollection). This form of questioning does not suggest 1 preferred experience, as single-item questionnaires might

Déjà vu is a subjective experience that may be nuanced in its differences from other experimentally generated experiences. If combined with a generalto-specific experimental experience questionnaire, this would provide an opportunity for participants to spontaneously report the occurrence of experimentally generated déjà vu

Differences between clinical and nonclinical experiences of déjà vu exist. Differences between experimentally generated and naturally occurring déjà vu experience, if found, may shed further light on the experience
Opportunity for comparison of experimentally generated déjà vu with naturally occurring déjà vu
No opportunity for comparison with naturally occurring déjà $\mathrm{vu}$
Responses are given as only "yes" or "no" 